INDO GLOBAL JOURNAL OF

PHARMACEUTICAL SCIENCES

ISSN 2249- 1023

\title{
Virtual Screening and ADMET Studies of Phytomolecules Targeting DNA Topoisomerase II
}

\author{
Yogesh Kumar $^{1}$, Feroz Khan ${ }^{1 *}$, Laiq-ur-Rahman ${ }^{2}$ \\ ${ }^{1}$ Metabolic and Structural Biology Dept., CSIR-Central Institute of Medicinal and Aromatic Plants, P.O.- CIMAP, Lucknow-226015
} (U.P.), India

${ }^{2}$ Biotechnology Division, CSIR-Central Institute of Medicinal and Aromatic Plants, P.O.- CIMAP, Lucknow -226015 (U.P.), India Address for Correspondance: Feroz Khan, f.khan@cimap.res.in

Keywords Phytomolecules; ADMET; DNA Topoisomerase II; Molecular Docking; Anti-cancer.

Conference Proceedings: International Conference on Advances in Plant and Microbial Biotechnology (PMB2017); JIIT, Noida: February 02-04, 2017

\begin{abstract}
Cancer is one of the most common, devastating class of disease affecting millions of people per year. Cancer has been estimated second leading cause of death in developing countries next to cardiovascular diseases. There are almost more than 100 types of cancer, each classified by the type of cell that is initially affected. In cancer, uncontrolled and abnormal growth of cells occurs with the potential to invade and spread to other parts of the body. Recent molecular studies have greatly expanded the study of most targeted gene, DNA topoisomerase II (TOP2), an enzyme that controls and alters the topological states of DNA during transcription. TOP2 became interesting cancer target for researchers, when the discovery of approved anticancer drugs, like Etoposide, doxorubicin, daunorubicin, amsacrine, amrubicin and many others, were held targeting TOP2. These drugs have toxic properties and have a most dangerous side effect like cardiomyopathy, leading to congestive heart failure. Due to these side effects and toxic effects of present drugs, we need to discover less or nontoxic compounds which inhibit the cancer. We performed molecular docking (virtual screening and eADMET) study of phytomolecules against most studied cancer target TOP2 to identify better as well as more potential compounds. More than 1000 of phytomolecules were docked againstTOP2 PDB ID: 1ZXM using FlexX docking software to identify the potential compounds, which inhibit the TOP2. For more authenticated results comparative docking studies were performed with targeted protein using. 8 approved and 5 investigational anti-cancer drugs targeting TOP2. Identified potential phytomolecules were screened based on their binding score higher than that of control drugs. These top 20 coded compounds from A1 to A20 were selected and finally a library of phytomolecules was prepared. ADMET properties of these phytomolecules were also calculated and compared with standard drugs, to know their pharmacological properties. These identified phytomolecules can be further investigated to know their inhibition property against TOP2 and to be drugs like molecules. (C) 2016 iGlobal Research and Publishing Foundation. All rights reserved.
\end{abstract}

Indo Global Journal of Pharmaceutical Sciences( ISSN 22491023 ; CODEN- IGJPAI; NLM ID: 101610675) indexed and abstracted in EMBASE(Elsevier), SCIRUS(Elsevier),CABI, CAB Abstracts, Chemical Abstract Services(CAS), American Chemical Society(ACS), Index Copernicus, EBSCO, DOAJ, Google Scholar and many more. For further details, visit http://iglobaljournal.com 